\title{
Clinical and Laboratory Characteristics and Outcome of Illness Caused by Tick-Borne Encephalitis Virus without Central Nervous System Involvement
}

\author{
Petra Bogovič, Andrej Kastrin, Stanka Lotrič-Furlan, Katarina Ogrinc, \\ Tatjana Avšič Županc, Miša Korva, Nataša Knap, Franc Strle
}

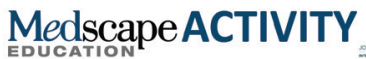

In support of improving patient care, this activity has been planned and implemented by Medscape, LLC and Emerging Infectious Diseases. Medscape, LLC is jointly accredited by the Accreditation Council for Continuing Medical Education (ACCME), the Accreditation Council for Pharmacy Education (ACPE), and the American Nurses Credentialing Center (ANCC), to provide continuing education for the healthcare team.

Medscape, LLC designates this Journal-based CME activity for a maximum of 1.00 AMA PRA Category 1 Credit(s) ${ }^{\mathrm{TM}}$. Physicians should claim only the credit commensurate with the extent of their participation in the activity.

Successful completion of this CME activity, which includes participation in the evaluation component, enables the participant to earn up to $1.0 \mathrm{MOC}$ points in the American Board of Internal Medicine's (ABIM) Maintenance of Certification (MOC) program. Participants will earn MOC points equivalent to the amount of CME credits claimed for the activity. It is the CME activity provider's responsibility to submit participant completion information to ACCME for the purpose of granting ABIM MOC credit.

All other clinicians completing this activity will be issued a certificate of participation. To participate in this journal CME activity: (1) review the learning objectives and author disclosures; (2) study the education content; (3) take the post-test with a 75\% minimum passing score and complete the evaluation at http://www.medscape.org/journal/eid; and (4) view/print certificate. For CME questions, see page $X X X$.
\end{abstract}

Release date: January 21, 2022; Expiration date: January 21, 2023

\section{Learning Objectives}

Upon completion of this activity, participants will be able to:

- Distinguish the typical disease course of tick-borne encephalitis virus

- Assess clinical characteristics of patients with tick-borne encephalitis virus

- Analyze trends in laboratory values among patients with tick-borne encephalitis virus

- Identify the rate of central nervous system involvement in the current case series of tick-borne encephalitis virus

CME Editor

Jill Russell, BA, Technical Writer/Editor, Emerging Infectious Diseases. Disclosure: Jill Russell, BA, has disclosed no relevant financial relationships.

\section{CME Author}

Charles P. Vega, MD, Health Sciences Clinical Professor of Family Medicine, University of California, Irvine School of Medicine, Irvine, California. Disclosure: Charles P. Vega, MD, has disclosed the following relevant financial relationships: served as an advisor or consultant for Johnson \& Johnson.

\section{Authors}

Petra Bogovič, MD, PhD; Andrej Kastrin, PhD; Stanka Lotrič-Furlan, MD, PhD; Katarina Ogrinc, MD, PhD; Tatjana Avšič Županc, PhD; Miša Korva, PhD; Nataša Knap, PhD; and Franc Strle, MD, PhD. 
Information on febrile illness caused by tick-borne encephalitis virus (TBEV) without central nervous system involvement is limited. We characterized 98 patients who had TBEV RNA in their blood but no central nervous system involvement at the time of evaluation. Median duration of illness was 7 days; $37(38 \%)$ patients were hospitalized. The most frequent findings were malaise or fatigue $(98 \%)$, fever $(97 \%)$, headache $(86 \%)$, and myalgias $(54 \%)$; common laboratory findings were leukopenia (88\%), thrombocytopenia (59\%), and abnormal liver test results $(63 \%)$. During the illness, blood leukocyte counts tended to improve, whereas thrombocytopenia and liver enzymes tended to deteriorate. At the time of positive PCR findings, 0/98 patients had serum IgG TBEV and 7 serum IgM TBEV; all patients later seroconverted. Viral RNA load was higher in patients with more severe illness but did not differ substantially in relation to several other factors. Illness progressed to tick-borne encephalitis in $84 \%$ of patients within 18 days after defervescence.

$\mathrm{T}$ ick-borne encephalitis virus (TBEV) is a member of the genus Flavivirus in the family Flaviviridae and is transmitted to humans predominantly through Ixodes spp. tick bites. In addition to 3 well-known subtypes of TBEV (European, Siberian, and Far Eastern) that cause disease in humans, other subtypes, including the currently named Baikalian and Himalayan subtypes, have been reported $(1,2)$.

Infection with TBEV can be symptomatic or asymptomatic. As is the case for infections with other flaviviruses, most $(70 \%-98 \%)$ persons infected with TBEV do not experience symptoms; however, some findings in blood donors suggest that asymptomatic infections might be rare (3). Nevertheless, when infection with TBEV is symptomatic, it can manifest as a febrile illness without central nervous system (CNS) involvement (Figure 1, panel A) but often progresses to tick-borne encephalitis (TBE) (i.e., CNS involvement caused by the virus) (Figure 1, panel B). Clinical manifestation differs in some respects according to virus subtype. In $13 \%-44 \%$ of patients, TBE caused by the European subtype manifests with direct CNS involvement (4-15) (Figure 1, panel C), whereas in most patients, CNS inflammation is preceded by a febrile illness, resulting in a biphasic course (Figure 1 , panel B). The initial phase, which corresponds to viremia, manifests as fever, fatigue, malaise, headache, and muscle and joint pain, but in the absence of CNS inflammation; this phase usually lasts $<1$ week (8), and the illness then improves over a few days. The hallmark of the second phase of the disease is CNS involvement. Meningitis is the predominant manifestation in children. In adults, meningitis occurs in $\approx 50 \%$ of patients, meningoencephalitis in $\approx 40 \%$, and meningoencephalomyelitis in $\approx 5 \%$ $10 \%$. The case-fatality rate of TBE caused by the European subtype of TBEV is $0.5 \%-2 \%$. In addition, $\approx 5 \%$ of adult patients are affected by permanent pareses, and at least one third suffer from a postencephalitic syndrome (16-19).

In general, clinical manifestations and laboratory characteristics of symptomatic TBEV infection are well described. However, this statement is valid for cases with neurologic involvement (i.e., for TBE) but less so for the initial phase of TBE, and much less so for TBEV infection manifesting solely as febrile illness without later CNS involvement. That manifestation, also called isolated initial phase of TBE, abortive form of TBE, febrile headache, summer flu, or fever form, is postulated to match clinically and serologically the initial phase of TBE, with the exception that subsequent CNS involvement does not occur. TBEV infection manifesting as febrile illness without later CNS involvement is suggested to be frequent (20-23), although not in all reports $(5,6,24,25)$, and the scientific basis for such a conclusion is unclear. Furthermore, although the outcome of symptomatic TBEV infection without CNS involvement is believed to be favorable, no reliable data on the outcome have been published. Because clinical symptoms and signs of the illness are nonspecific, and because, in parallel to the initial phase of TBE, serum antibodies to TBEV are not yet expected to have developed, the only option for diagnosis at the time of actual illness is demonstrating the presence of TBEV RNA in the blood. However, this approach is not routine and might have a low diagnostic yield owing to several other known or unknown causes of fever, even in a region that is highly endemic for TBE. Therefore, the possibility that a febrile illness is the result of TBEV infection is usually tested for and established only after signs or symptoms of CNS involvement appear, which does not happen in case of the fever form. In that case (and if PCR detection of viral RNA in blood is not available), further clinical and microbiologic (serologic) follow-up after improvement is needed to establish the diagnosis. In this study, we analyzed in detail the clinical and laboratory characteristics of febrile illness after tick bite or exposure to ticks and its outcome in patients in whom infection with TBEV was established by the presence of viral RNA in the blood.

\section{Materials and Methods}

\section{Definitions}

Febrile illness resulting from infection with TBEV was defined by the presence of fever and constitutional symptoms, demonstration of viral RNA 


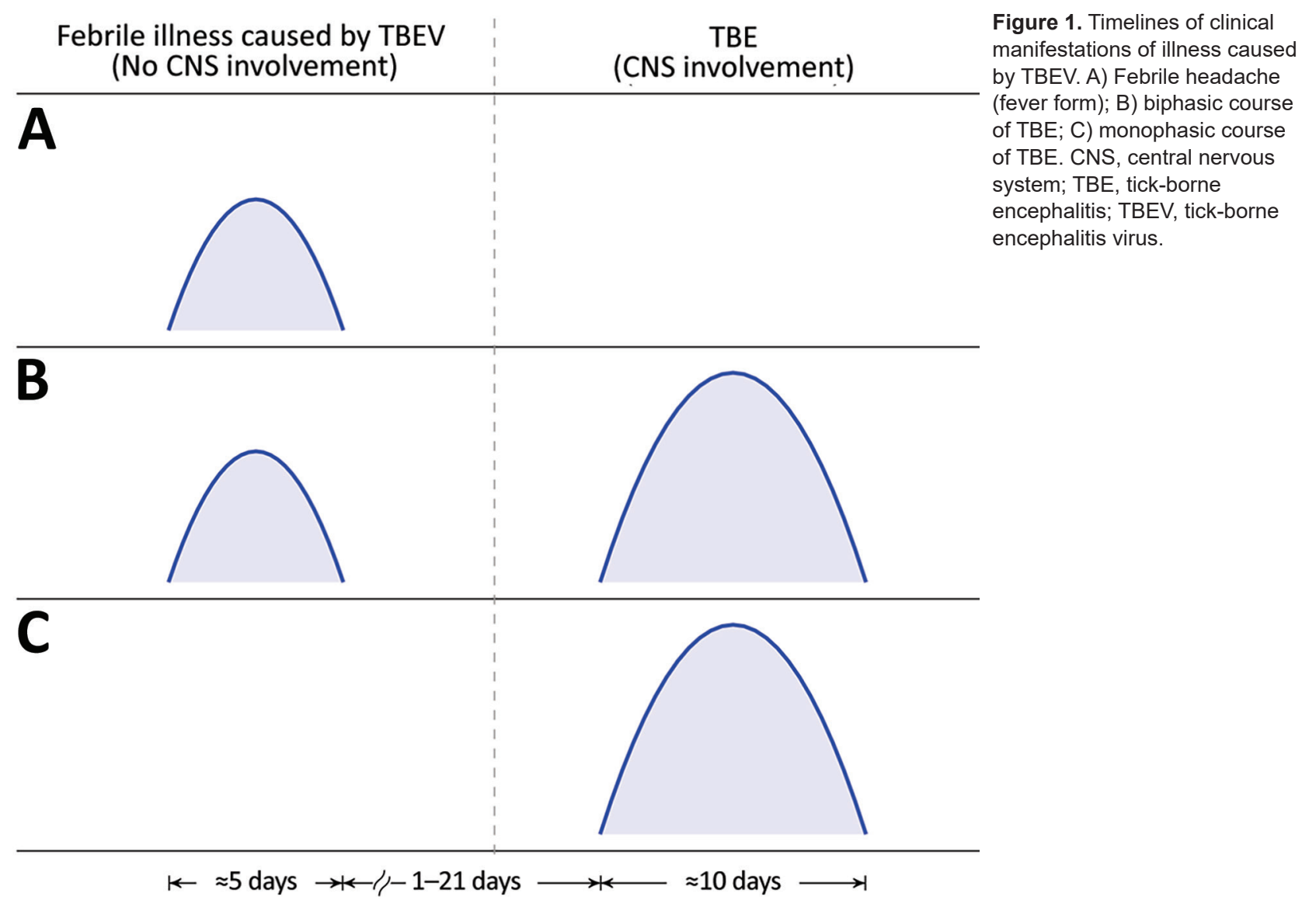

in serum specimens, and the absence of signs or symptoms of CNS involvement at the time of illness. In patients with clinical signs potentially suggesting CNS involvement, cerebrospinal fluid (CSF) samples were examined; the threshold for lumbar puncture was low. A CSF leukocyte count $\leq 5 \times 10^{6} / \mathrm{L}$ was interpreted as excluding CNS inflammation.

According to the later appearance (or absence) of neurologic involvement, the febrile illness was further subclassified as either the initial phase of TBE (defined as a febrile illness with demonstration of viral RNA in serum samples that, after a clinical improvement, was followed by neurologic involvement within a 2-month follow-up period and fulfilling criteria for TBE) or as febrile illness resulting from infection with TBEV in a narrow sense (fever form, febrile headache) when no signs of CNS involvement were present at the time of actual illness or within a 2-month follow-up period. TBE was defined as the presence of clinical signs or symptoms of meningitis or meningoencephalitis, increased CSF leukocyte counts $\left(>5 \times 10^{6}\right.$ cells/L), and demonstration of a recent infection with TBEV indicated by serum IgM and IgG or IgG seroconversion in paired serum samples.

\section{Patients and Samples}

Adult patients examined for febrile illness at the Department of Infectious Diseases, University Medical Center Ljubljana (Ljubljana, Slovenia), during 20032019, in whom the presence of TBEV RNA was identified by PCR in serum specimens, qualified for the study. Serum samples were obtained either during a prospective study on the etiology of febrile illness after a tick bite or exposure to ticks (62 patients, 63.3\%) or represented remnants of samples collected as a part of routine diagnostic testing of patients with febrile illness in whom TBE later occurred (36 patients, 36.7\%). Serum specimens were stored at $-80^{\circ} \mathrm{C}$ until further processing. For the 62 patients, we obtained clinical and laboratory information on the etiology of febrile illness occurring after tick bite or tick exposure prospectively. Clinical and laboratory follow-up occurred for these patients for at least 2 months (i.e., at first evaluation and at follow-up visits 1 week, 2 weeks, and 2 months later). For the other 36 patients, we obtained clinical and laboratory information from medical charts. 


\section{TBEV Antibodies and RNA Load}

We determined the presence of TBEV antibodies in serum samples by using the Enzygnost Anti-TBE/ FSME Virus (IgM, IgG) test (Siemens AG, https:// www.siemens.com), according to the manufacturer's instructions. We extracted total RNA from serum samples by using the QIAamp Viral RNA Mini Kit (QIAGEN, https://www.qiagen.com), according to the manufacturer's instructions. For the detection of TBEV RNA, we performed quantitative reverse transcription PCR as reported previously (26).

\section{Statistical Analysis}

We summarized continuous variables as median values and interquartile ranges (IQRs), and discrete variables as counts and percentages with 95\% CIs. We based comparisons between groups on Wilcoxon rank-sum tests for continuous variables and Fisher exact tests for discrete variables. We defined statistical significance as a $\mathrm{p}$ value of $\leq 0.05$.

We examined associations between variables by using linear regression modeling (Figures 2, 3). We used $\log _{10}$-transformed viral RNA loads, and domain experts (P.B. and F.S.) selected included covariates. We modeled continuous covariates that demonstrated a nonlinear relationship by using restricted cubic splines (27) and imputed missing values by using multiple imputation on the basis of additive regression, bootstrapping, and predictive mean matching (28). We used R software for all statistical analyses (29).

\section{Ethics}

The study was conducted in accordance with the principles of the Declaration of Helsinki, the Oviedo Convention on Human Rights and Biomedicine, and the Slovene Code of Medical Deontology. The study was approved by the National Medical Ethics Committee of Slovenia (approval nos. 152/06/13, 178/02/13, and $37 / 12 / 13)$. Patients whose specimens were obtained in the study on the etiology of febrile illness after a tick bite or exposure to ticks signed an informed consent form. The Ethics Committee waived the need for written informed consent for patients for whom remnants of routinely collected serum specimens were used.

\section{Results}

A total of 98 adult patients examined for febrile illness in whom TBEV RNA was identified by PCR in their serum specimens were enrolled in the study. The median age of the patients was 51 years; $52 \%$ were women.

\section{Clinical and Laboratory Characteristics of Febrile IIIness Caused by TBEV}

Most $(88.7 \%)$ patients reported a tick bite within 4 weeks of the onset of illness. The median time from the bite to illness onset was 6 days, median duration of illness before evaluation was 5 days, and total duration of the illness was 7 days. A total of $37 / 98(37.8 \%)$ patients were hospitalized for a median of 3 days. The most frequent symptoms or signs were malaise and fatigue $(98 \%)$, fever $(96.9 \%)$, headache $(85.7 \%)$, and myalgias $(54.1 \%)$ (Table 1$)$. The most frequent laboratory findings were leukopenia $(87.5 \%)$, thrombocytopenia (59.4\%), and abnormal liver test results $(62.5 \%$ of patients had $\geq 1$ abnormal liver test result, most often elevated aspartate aminotransferase [AST, 55.0\%] and alanine aminotransferase [ALT, 26.3\%]) (Table 2).

Individual laboratory parameters according to duration of illness before testing demonstrated heterogeneous results. Counts of total peripheral blood leukocytes, neutrophils, lymphocytes, and monocytes were lowest early in the course of illness and tended to increase (improve) with the duration of illness, whereas thrombocytopenia became more pronounced with the duration of illness. Liver tests, including AST, ALT, gamma-glutamyl transferase (GGT), and lactate dehydrogenase, also tended to deteriorate with the duration of illness. These tendencies were noticeably uniform and were significant for total leukocyte and monocyte counts and for AST, ALT, and GGT levels (Figure 2).

None of the 98 patients had serum IgG to TBEV at the time of a positive PCR result (median of 5 days after illness onset) and only 2/98 (2.0\%) had TBEVspecific IgM. In these 2 patients, the serologic tests were performed on days 7 and 13 of the illness. An additional 5 patients $(5.1 \%)$ had borderline specific IgM levels; for most, the duration of illness before testing was somewhat longer (median 6 days, range 3-9 days). Viral RNA load was higher in hospitalized patients with more severe illness than in those who did not need hospitalization but did not differ substantially according to age, sex, duration of illness before testing, or total duration of the actual febrile illness, or for patients with undetectable viral IgM in serum samples when compared with patients in whom antibodies were detectable (Figure 3).

\section{Outcome}

Of the 62 patients who received a diagnosis during a prospective study on the etiology of febrile illness after a tick bite or exposure to ticks and in whom TBEV was present in serum samples, $6(9.7 \%)$ did not 

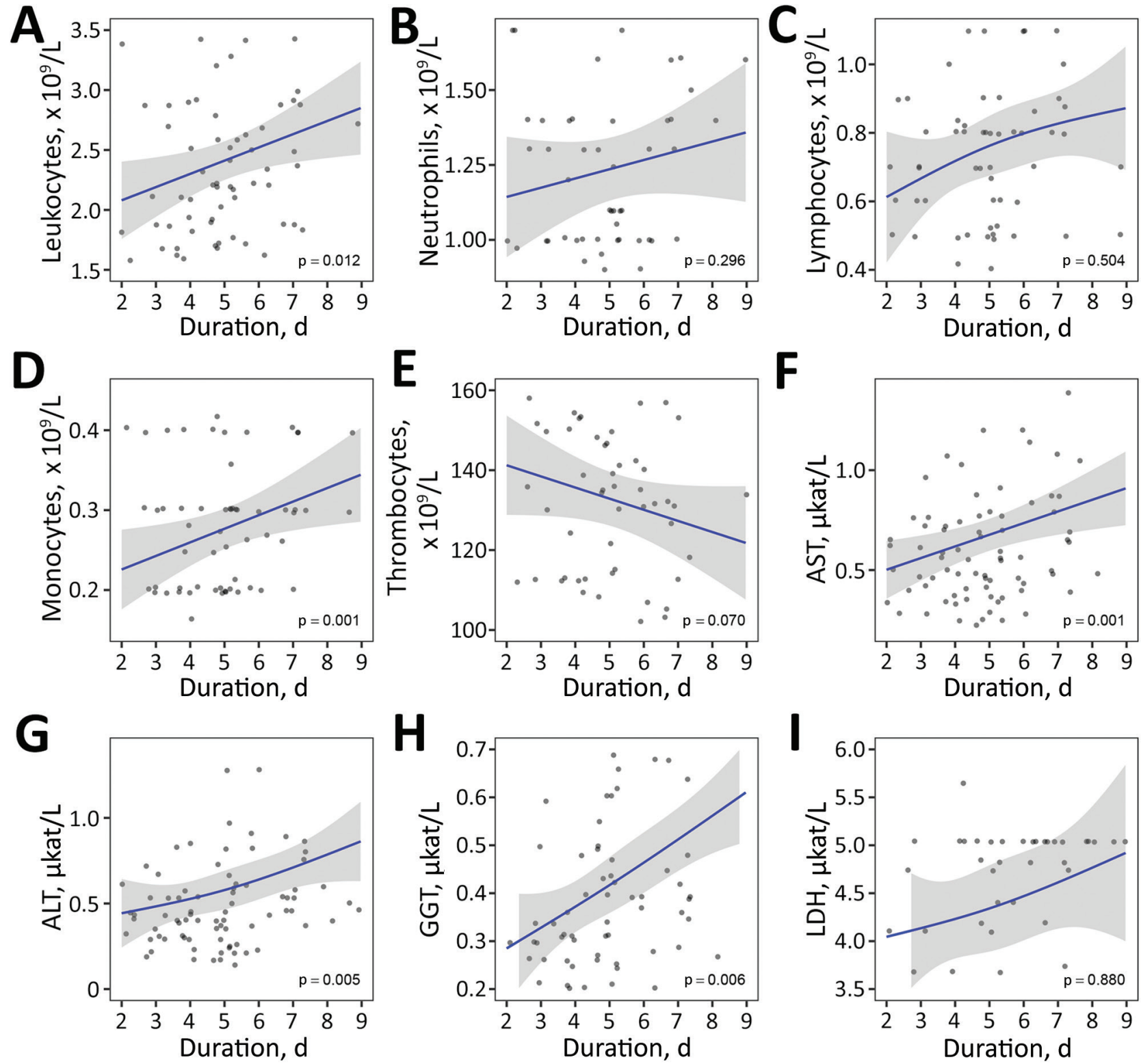

Figure 2. Laboratory findings according to illness duration in cases of febrile illness caused by tick-borne encephalitis virus without central nervous system involvement at the time of evaluations, Slovenia. A) Leukocytes, B) neutrophils, C) lymphocytes, D) monocytes, E) thrombocytes, F) AST, G) ALT, H) GGT, and I) LDH. Blue lines indicate loess regression lines; shaded areas indicate $95 \%$ Cls. Relationships between variables in panels C, G, and I were modeled by using restricted cubic splines with 3 knots (25). ALT, alanine aminotransferase; AST, aspartate aminotransferase; GGT, gamma-glutamyl transferase; LDH, lactate dehydrogenase.

experience any symptoms or signs during the followup period of 2 months. In 4 (4.5\%) patients, mild constitutional symptoms not suggesting CNS involvement and without meningeal signs reappeared after a symptom-free interval of up to 12 days. In contrast, the other $52(83.9 \%)$ patients experienced overt signs of meningitis or meningoencephalitis associated with CSF pleocytosis and fulfilled serologic criteria for TBE; in this subgroup, the longest symptom-free interval was 18 days.
The clinical characteristics of the initial phase of TBE and febrile illness without subsequent CNS involvement were not formally compared because the number of patients was too small, but the clinical and laboratory manifestation of the 2 entities appears comparable (Table 3). All patients developed IgM and IgG to the virus during follow-up.

Of the 62 prospectively followed patients, 27 $(43.5 \%)$ were hospitalized. The likelihood of later CNS involvement in hospitalized patients was similar to that 

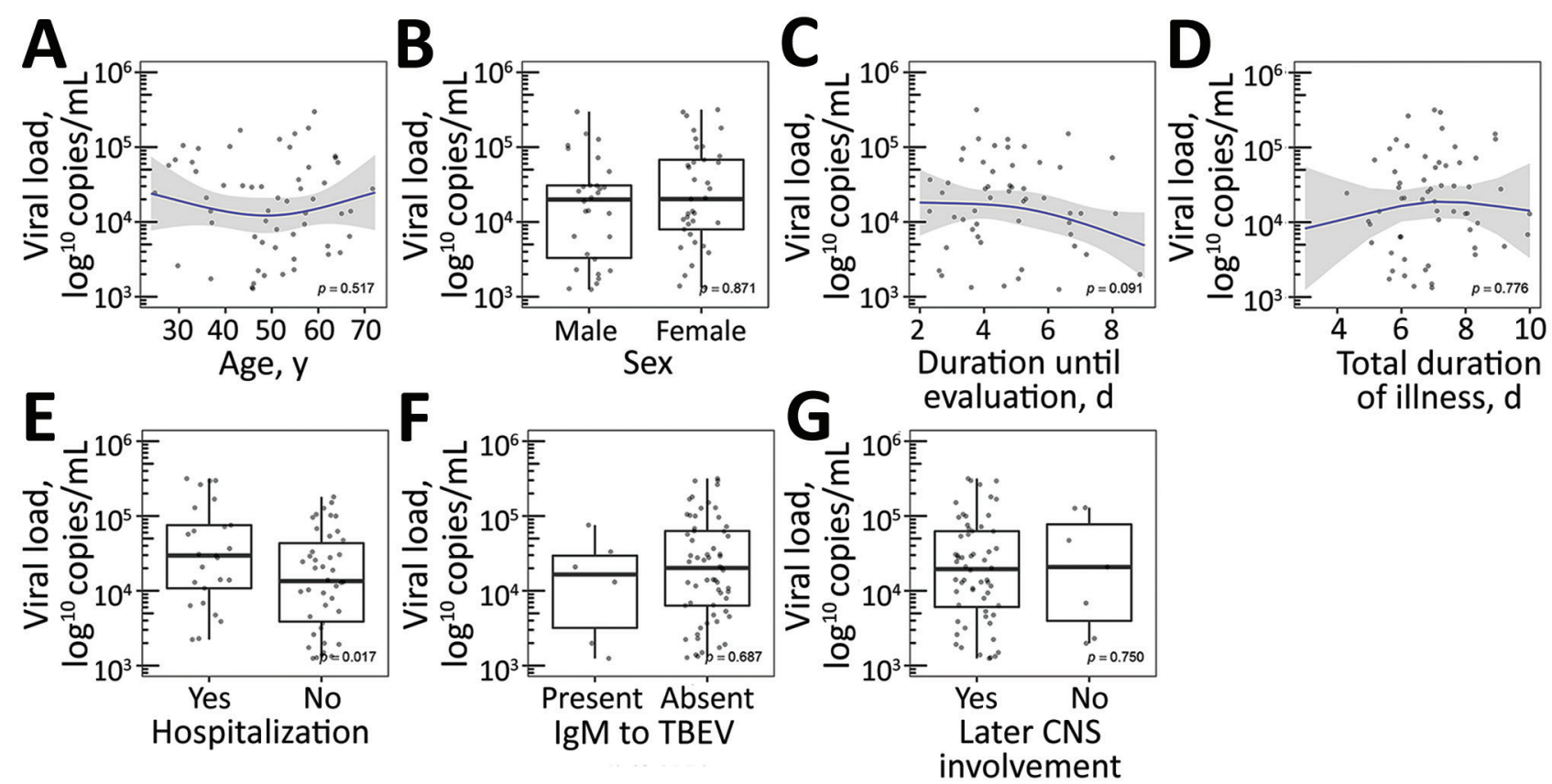

Figure 3. TBEV RNA load according to demographic and clinical characteristics in cases of febrile illness without central nervous system involvement at the time of evaluations, Slovenia. A) Age, B) sex, C) duration of illness until evaluation, D) total duration of illness, E) hospitalization, F) presence of IgM to TBEV, and G) later CNS involvement. Blue lines indicate loess regression lines; shaded areas indicate $95 \% \mathrm{Cls}$. Relationships between variables in panels panels $\mathrm{A}, \mathrm{C}$, and D are modeled by using restricted cubic splines with 3 knots (25). Comparisons between groups in panels B, E, F, and G are based on a Wilcoxon rank-sum test. CNS, central nervous system; TBEV, tick-borne encephalitis virus.

in patients with less severe illness who were treated as outpatients $(24 / 27$ [88.9\%] vs. $28 / 35$ [80\%]; $p=0.49)$. Furthermore, the level of viral RNA in serum samples in the group in which no CNS involvement occurred was similar to the level in those in whom meningitis or meningoencephalitis later occurred (Figure 3).

\section{Discussion}

Symptomatic infection with TBEV can manifest as CNS involvement (TBE) or as a febrile illness with or without subsequent CNS involvement (Figure 1). Although the epidemiology and clinical manifestation of symptomatic TBEV infection is considered well-established, this statement is valid only for neurologic involvement (TBE) and is less well-established for the initial phase of TBE and even less so for TBEV infection manifesting solely as febrile illness without later CNS involvement.

TBE is highly endemic to Slovenia. For as many as $70 \%$ of patients in Slovenia who have notified cases of TBE, diagnosis occurs at University Medical Center Ljubljana $(30,31)$, which provided access to detailed information on a large number of patients with TBE and enabled this study.

The initial phase of TBE consists of fever, headache, myalgias, arthralgias, and fatigue $(5,6,8,9,13,32$ -
34), but reliable information on the relative frequency of individual symptoms is limited, and results are variable. In this study, which encompasses TBEV febrile illness with and without later CNS involvement (the initial phase of TBE and febrile headache) and is based on a well-defined group of patients with definite proof of TBEV infection (the presence of virus in blood at the time of actual illness and later seroconversion), our findings corroborate previous results on the spectrum of symptom manifestation and add reliable information on their relative frequency. Thus, $>85 \%$ of patients have malaise or fatigue, fever, and headache; $\approx 50 \%$ of the patients report myalgias, arthralgias, and, rather unexpectedly, gastrointestinal symptoms (abdominal pain, nausea, vomiting, or diarrhea); and the frequency of respiratory symptoms or chills is almost $20 \%$. The finding of chills is somewhat surprising because chills are not common in patients with viral infections and are more characteristic of diseases caused by bacteria. Illness duration in our patients (median 7 days) was somewhat longer than reported in the literature (median 4-6, range 1-19 days) $(5,6,8,9,12,26,32,34)$. In addition, in some patients, the illness was relatively severe: more than one third of patients were hospitalized. Laboratory findings possibly contributed to decisions to hospitalize 
because leukopenia and thrombocytopenia might suggest serious disease in a patient with fever.

The first phase of TBE is known to be accompanied by leukopenia; thrombocytopenia and abnormal liver test results also might be present, although to a lesser extent $(35,36)$. In contrast, in the second phase of TBE, the blood leukocyte count is elevated or in normal range. Laboratory tests in the patients in our study very often demonstrated abnormalities (leukopenia in $88 \%$, abnormal liver tests in $70 \%$, and thrombocytopenia in 59\%) that were more common than previously described $(6,13,25,26,35,36)$. Our study corroborates previous findings that in most patients, the concentration of total leukocytes in the peripheral blood is reduced. In addition, we offer several new findings, such as a reduction in all major subgroups of leukocytes and a tendency for total numbers of leukocytes, neutrophils, lymphocytes, and monocytes to increase (i.e., improve). In contrast, thrombocytopenia, liver tests (including AST, ALT, and GGT), and lactate dehydrogenase tended to deteriorate with duration of illness. Although we do not have an exact explanation for these laboratory abnormalities, they seem biologically plausible. During illness caused by
TBEV without CNS involvement (including illness with subsequent CNS involvement), TBEV replicates in various organs and tissues, and this might affect test results. Later, however, when viremia vanishes and CNS damage occurs, the abnormalities are not present $(7,8,16-18)$ and obviously are temporally associated with viremia, suggesting a direct or indirect effect of the virus on the bone marrow and liver.

Our results corroborate previous findings $(24,26,37)$ that the appearance of antibodies to TBEV greatly diminishes the likelihood of detecting viral RNA in blood: none of our 98 patients had detectable serum IgG to TBEV at the time of a positive PCR finding, although $7(7.6 \%)$ patients had specific IgM. As expected, in these 7 patients, the duration of illness was longer than for patients who were completely seronegative. We also expected that the viral RNA load would be lower in patients with detectable serum IgM to the virus than in patients in whom the antibodies were undetectable, but we did not confirm this premise. Furthermore, viral RNA load did not differ substantially in relation to age, sex, duration of illness before testing, or total duration of the actual febrile illness; however, viral RNA load was higher

\begin{tabular}{|c|c|}
\hline Characteristic & Value \\
\hline \multicolumn{2}{|l|}{ Sex } \\
\hline $\mathrm{F}$ & $51(52.0,41.7-62.2)$ \\
\hline M & $47(48.0,37.8-58.3)$ \\
\hline Median age, y (IQR) & $51(37-60.75)$ \\
\hline Underlying illnesses & $38(38.8,29.1-49.2)$ \\
\hline History of tick bite† & $86(88.7,80.6-94.2)$ \\
\hline Median incubation period, $\mathrm{d}(\mathrm{IQR}) \ddagger$ & $6(4-9.75)$ \\
\hline Median duration of illness before first evaluation, $d(I Q R)$ & $5(4-6)$ \\
\hline \multicolumn{2}{|l|}{ Clinical manifestation } \\
\hline Body temperature $\geq 37.5^{\circ} \mathrm{C}$ & $95(96.9,91.3-99.4)$ \\
\hline Median body temperature, ${ }^{\circ} \mathrm{C}(\mathrm{IQR}) \S$ & $38.3(37.8-38.9)$ \\
\hline Chills & $19(19.4,12.1-28.6)$ \\
\hline Headache & $84(85.7,77.2-92.0)$ \\
\hline Myalgia & $53(54.1,43.7-64.2)$ \\
\hline Arthralgia & $42(42.9,32.9-53.3)$ \\
\hline Gastrointestinal symptoms & $45(45.9,35.8-56.3)$ \\
\hline Abdominal pain & $2(2.0,0.3-7.2)$ \\
\hline Nausea, vomiting & $37(37.8,28.2-48.1)$ \\
\hline Diarrhea & $16(16.3,9.6-25.2)$ \\
\hline Malaise and fatigue & $96(98.0,92.8-99.8)$ \\
\hline Respiratory symptoms & $18(18.4,11.3-27.5)$ \\
\hline Sore throat & $11(11.2,5.7-19.2)$ \\
\hline Cough & $10(10.2,5.0-18.0)$ \\
\hline Median duration of illness, initial phase, $d(I Q R) \pi$ & $7(6-8)$ \\
\hline \multicolumn{2}{|l|}{ Hospitalization } \\
\hline No. hospitalized patients & $37(37.8,28.2-48.1)$ \\
\hline Median duration of hospitalization, $d(I Q R) \#$ & $3(1-5)$ \\
\hline \multicolumn{2}{|c|}{$\begin{array}{l}\text { *Values are no. (\%, } 95 \% \mathrm{Cl}) \text { except as indicated. IQR, interquartile range. } \\
\text { TTick bite within } 4 \text { weeks before onset of illness. Data available for } 97 \text { patients. } \\
\text { †Time (days) from tick bite to onset of illness calculated only in patients with } 1 \text { bite. Data available for } 54 \text { patients. } \\
\text { §Highest temperature in the course of the illness. } \\
\text { TData available for } 82 \text { patients. } \\
\text { \#Data available for } 33 \text { patients. }\end{array}$} \\
\hline
\end{tabular}


Table 2. Laboratory data on adult patients who had febrile illness caused by tick-borne encephalitis virus without central nervous system involvement at the time of evaluation, Slovenia*

\begin{tabular}{|c|c|}
\hline Laboratory findings & Value \\
\hline Median blood leukocyte count, $\times 10^{9} / \mathrm{L}$ & $2.3(1.8-3.125)$ \\
\hline$(\mathrm{IQR}) \dagger$ & \\
\hline Blood leukocyte count $<4 \times 10^{9} / \mathrm{L}$ & $84(87.5,79.2-93.4)$ \\
\hline Blood leukocyte count $>10 \times 10^{9} / \mathrm{L}$ & $0(0,0-3.8)$ \\
\hline Median blood neutrophil count, $\times 10^{9} / \mathrm{L}$ & $1.22(0.9-1.7)$ \\
\hline$(I Q R) \ddagger$ & \\
\hline Blood neutrophil count $<1.5 \times 10^{9} / \mathrm{L}$ & $55(65.5,54.3-75.5)$ \\
\hline Blood neutrophil count $>7.4 \times 10^{9} / \mathrm{L}$ & $0(0,0-4.3)$ \\
\hline Median blood lymphocyte count, $\times 10^{9} / \mathrm{L}$ & $0.88(0.5-1.1)$ \\
\hline$(I Q R) \ddagger$ & \\
\hline Blood lymphocyte count $<1.1 \times 10^{9} / \mathrm{L}$ & $61(72.6,61.8-81.8)$ \\
\hline Blood lymphocyte count $>3.5 \times 10^{9} / \mathrm{L}$ & $1(1.2,0-6.5)$ \\
\hline Median blood monocyte count, $\times 10^{9} / \mathrm{L}$ & $0.29(0.2-0.4)$ \\
\hline (IQR)‡ & \\
\hline Blood monocyte count $<0.21 \times 10^{9} / \mathrm{L}$ & $34(40.5,29.9-51.8)$ \\
\hline Blood monocyte count $>0.92 \times 10^{9} / \mathrm{L}$ & $0(0,0-4.3)$ \\
\hline Median blood platelet count, $\times 10^{9} / \mathrm{L}(\mathrm{IQR}) \dagger$ & $132(110.5-157)$ \\
\hline Blood platelet count $<140 \times 10^{9} / \mathrm{L}$ & $57(59.4,48.9-69.3)$ \\
\hline Serum C-reactive protein level $>5 \mathrm{mg} / \mathrm{L} \uparrow \S$ & $9(9.4,4.4-17.1)$ \\
\hline Liver test results, $\mu \mathrm{kat} / \mathrm{L}(\mathrm{IQR}) \mathrm{T}$ & \\
\hline Median alkaline phosphatase & $0.93(0.755-1.06)$ \\
\hline Elevated $>1.92$ & $0(0,0-4.6)$ \\
\hline Median aspartate aminotransferase\# & $0.615(0.4575-0.8325)$ \\
\hline Elevated $>0.58$ & $44(55.0,43.5-66.2)$ \\
\hline Median alanine aminotransferase\# & $0.53(0.37-0.805)$ \\
\hline Elevated $>0.77$ & $21(26.3,17.0-37.3)$ \\
\hline Median gamma-glutamyl transferaseף & $0.37(0.265-0.595)$ \\
\hline Elevated $>0.92$ & $9(11.4,5.3-20.5)$ \\
\hline Median lactate dehydrogenase ${ }^{\star *}$ & $3.48(2.92-4.82)$ \\
\hline Elevated $>4.13$ & $12(36.4,20.4-54.9)$ \\
\hline $\begin{array}{l}\text { No. patients with } \geq 1 \text { abnormal liver test } \\
\text { result } † \dagger\end{array}$ & $50(62.5,51.0-73.1)$ \\
\hline $\begin{array}{l}\text { *Values are no. (\%, } 95 \% \mathrm{Cl}) \text { unless otherwise } \\
\text { †Data available for } 96 \text { patients. } \\
\text { †Data available for } 84 \text { patients. } \\
\text { §Median } 7 \text {, range } 6-45 \text {. } \\
\text { TData available for } 79 \text { patients. } \\
\text { \#Data available for } 80 \text { patients. } \\
\text { **Data available for } 33 \text { patients. } \\
\text { ††Data available for } 80 \text { patients. }\end{array}$ & \\
\hline
\end{tabular}

in patients with more severe illness (those who were hospitalized compared with those who were not). Information on TBEV RNA load in humans is very limited $(24,26)$. In our previous report on viral RNA load in patients with biphasic course of TBE, the load was higher in women than in men but was not significantly associated with clinical and laboratory characteristics of the initial phase of illness or with characteristics of the later meningoencephalitic phase of TBE (26). However, because several associations tested in this study were not analyzed in our previous study (and vice versa), direct comparison is restricted to matching approaches; within them, the only discordant result was for viral RNA load according to sex, which was significantly higher in women than men in the previous study but not in this study.

Some review articles have stated that febrile illness without later CNS involvement is a frequent clinical manifestation of infection with TBEV, representing approximately two thirds of all clinically manifested infections with TBEV (20-23). However, a PubMed literature search of titles and abstracts using the search terms "(tick-borne encephalitis) AND (initial phase OR first phase)" and without time limitation (i.e., from 1966 onward) did not reveal any primary source firmly supporting such statements for Western and Central Europe. Nevertheless, febrile illness resulting from TBEV infection without subsequent CNS involvement is usually not recognized, possibly because of the small proportion of such cases (38), which is in accordance with some other reports $(5,6,24,25)$. Our previous findings, which were based on clinical and serologic analyses of febrile illness after a tick bite, suggested that febrile illness resulting from infection with TBEV occurs as a rule with subsequent CNS involvement (TBE) after an improvement of up 
to 12 days $(25,34)$. In this study, which was focused on 62 patients with well-defined TBEV infection, 52 (84\%) patients experienced overt symptoms and signs of CNS involvement associated with CSF lymphocyt- ic pleocytosis, $6(10 \%)$ patients remained completely asymptomatic, and $4(6 \%)$ patients in whom CSF was not examined experienced mild symptoms not associated with meningeal signs for a duration of 2-7 days.

Table 3. Basic demographic, clinical, and laboratory characteristics of febrile illness caused by tick-borne encephalitis virus infection with or without subsequent central nervous system involvement*

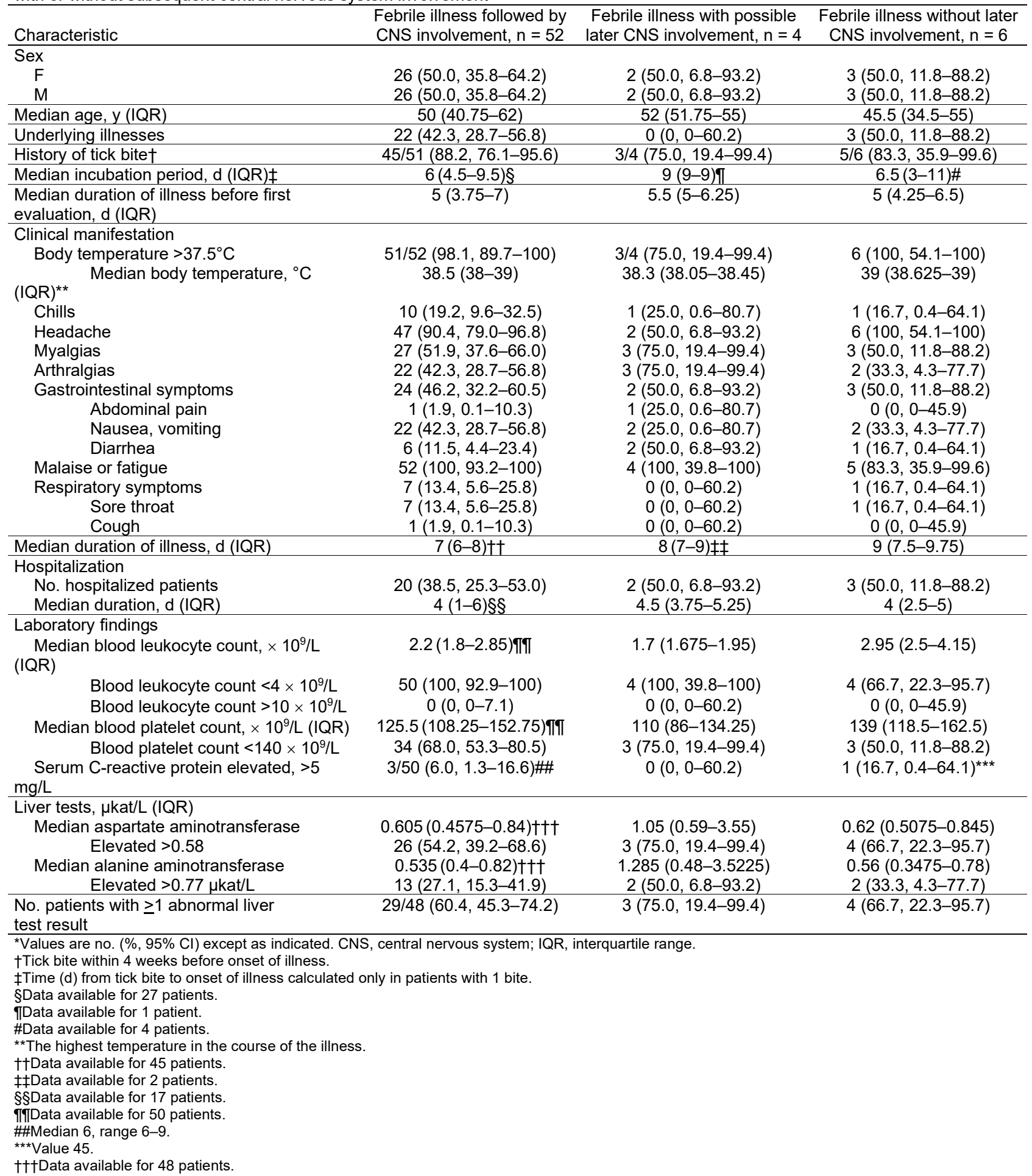


We expected that patients in whom TBE did not occur would have lower blood levels of virus than those in whom CNS involvement occurred; these speculations were not confirmed.

The main strengths of our study are the sufficiently large number of patients with a well-defined diagnosis of febrile illness resulting from TBEV infection (on the basis of demonstration of viral RNA in serum samples during actual illness and on later seroconversion), together with the comprehensive collection of clinical and laboratory data and assessment of the course and outcome of the illness. We reveal several new clinical and laboratory details of febrile illness caused by TBEV, confirming that in most (at least $84 \%$ ) of these patients, TBE (i.e., CNS inflammation) later develops. However, because we included only patients referred to us by family physicians, one of the limitations of the study is a potential selection bias: patients with very mild illness probably do not visit primary physicians and, even if they do, the likelihood of referral to us is lower than for patients with more severe disease or unusual laboratory findings, such as leukopenia or thrombocytopenia. Thus, our findings are limited to a subset of patients with more severe disease; consequently, our conclusions might not be valid for milder clinical cases.

In conclusion, febrile illness caused by TBEV infection is characterized clinically by the presence of malaise or fatigue (98\%), fever (97\%), headache (86\%), and myalgias (54\%) and in laboratory tests by leukopenia (88\%), thrombocytopenia (59\%), and abnormal liver test results $(63 \%$ of patients had $\geq 1$ abnormal liver test, usually elevated AST and ALT) but normal inflammatory markers. The infection proceeded to TBE in $\geq 5 / 6(84 \%)$ patients within 18 days after defervescence. Clinical and laboratory findings in patients with TBEV febrile illness do not distinguish between patients in whom TBE later develops and those in whom it does not.

\section{About the Author}

Dr. Bogovič is an infectious diseases specialist in the Department of Infectious Diseases of the University Medical Center Ljubljana, Ljubljana, Slovenia. Her primary research interests are zoonoses, particularly tick-transmitted diseases.

\section{References}

1. Demina TV, Dzhioev YP, Verkhozina MM, Kozlova IV, Tkachev SE, Plyusnin A, et al. Genotyping and characterization of the geographical distribution of tick-borne encephalitis virus variants with a set of molecular probes. J Med Virol. 2010;82:965-76. https://doi.org/10.1002/jmv.21765
2. Kozlova IV, Demina TV, Tkachev SE, Doroschenko EK, Lisak OV, Verkhozina MM, et al. Characteristics of the Baikal subtype of tick-borne encephalitis virus circulating in Eastern Siberia. Acta Biomedicia Scientifica. 2018;3:53-60. https://doi.org/10.29413/ABS.2018-3.4.9

3. Marvik $\AA$, Tveten Y, Pedersen AB, Stiasny K, Andreassen $\AA$ K, Grude N. Low prevalence of tick-borne encephalitis virus antibodies in Norwegian blood donors. Infect Dis (Lond). 2021;53:44-51. https:/ / doi.org/10.1080/23744235.2020. 1819561

4. Tomažič J, Pikelj F, Schwartz B, Kunze M, Kraigher A, Matjašič M, et al.; Slovenian TBE study group. The clinical features of tick-borne encephalitis in Slovenia. A study of 492 cases in 1994. Antibiot Monitor. 1996;12:115-20.

5. Günther G, Haglund M, Lindquist L, Forsgren M, Sköldenberg B. Tick-bone encephalitis in Sweden in relation to aseptic meningo-encephalitis of other etiology: a prospective study of clinical course and outcome. J Neurol. 1997;244:230-8. https://doi.org/10.1007/s004150050077

6. Kaiser R. The clinical and epidemiological profile of tick-borne encephalitis in southern Germany 1994-98: a prospective study of 656 patients. Brain. 1999;122:2067-78. https:/ / doi.org/10.1093/brain/122.11.2067

7. Kaiser R, Holzmann H. Laboratory findings in tick-borne encephalitis - correlation with clinical outcome. Infection. 2000;28:78-84. https://doi.org/10.1007/s150100050051

8. Mickiene A, Laiskonis A, Günther G, Vene S, Lundkvist A, Lindquist L. Tickborne encephalitis in an area of high endemicity in lithuania: disease severity and long-term prognosis. Clin Infect Dis. 2002;35:650-8. https:/ / doi.org/ 10.1086/342059

9. Kaiser R. Tick-borne encephalitis (TBE) in Germany and clinical course of the disease. Int J Med Microbiol. 2002;291(Suppl 33):58-61. https:/ / doi.org/10.1016/ S1438-4221(02)80012-1

10. Logar M, Bogovic P, Cerar D, Avsic-Zupanc T, Strle F. Tick-borne encephalitis in Slovenia from 2000 to 2004: comparison of the course in adult and elderly patients. Wien Klin Wochenschr. 2006;118:702-7. https://doi.org/10.1007/ s00508-006-0699-6

11. Misić Majerus L, Daković Rode O, Ruzić Sabljić E. Postencephalitic syndrome in patients with tick-borne encephalitis [in Croatian]. Acta Med Croatica. 2009;63:269-78.

12. Radzišauskienė D, Urbonienè J, Kaubrys G, Andruškevičius S, Jatužis D, Matulytė E, et al. The epidemiology, clinical presentation, and predictors of severe tick-borne encephalitis in Lithuania, a highly endemic country: A retrospective study of 1040 patients. PLoS One. 2020;15:e0241587. https://doi.org/10.1371/journal.pone.0241587

13. Barp N, Trentini A, Di Nuzzo M, Mondardini V, Francavilla E, Contini C. Clinical and laboratory findings in tick-borne encephalitis virus infection. Parasite Epidemiol Control. 2020;10:e00160. https://doi.org/10.1016/j.parepi.2020.e00160

14. Bogovič P, Lotrič-Furlan S, Avšič-Županc T, Korva M, Kastrin A, Lusa L, et al. Comparison of clinical, laboratory and immune characteristics of the monophasic and biphasic course of tick-borne encephalitis. Microorganisms. 2021;9:796. https:/ / doi.org/10.3390/ microorganisms9040796

15. Kohlmaier B, Schweintzger NA, Sagmeister MG, Švendová V, Kohlfürst DS, Sonnleitner A, et al.; The Eu-Tick-Bo Study Group. Clinical characteristics of patients with tick-borne encephalitis (TBE): a European multicentre study from 2010 to 2017. Microorganisms. 2021;9:1420. https:/ / doi.org/ 10.3390/microorganisms 9071420

16. Mansfield KL, Johnson N, Phipps LP, Stephenson JR, Fooks AR, Solomon T. Tick-borne encephalitis virus - 
a review of an emerging zoonosis. J Gen Virol. 2009;90:178194. https://doi.org/10.1099/vir.0.011437-0

17. Bogovic P, Strle F. Tick-borne encephalitis: a review of epidemiology, clinical characteristics, and management. World J Clin Cases. 2015;3:430-41. https:/ / doi.org/ 10.12998/wjcc.v3.i5.430

18. Taba P, Schmutzhard E, Forsberg P, Lutsar I, Ljøstad U, Mygland $\AA$, et al. EAN consensus review on prevention, diagnosis and management of tick-borne encephalitis. Eur J Neurol. 2017;24:1214-e61. https:/ / doi.org/10.1111/ ene.13356

19. Bogovič P, Stupica D, Rojko T, Lotrič-Furlan S, Avšič-Županc T, Kastrin A, et al. The long-term outcome of tick-borne encephalitis in Central Europe. Ticks Tick Borne Dis. 2018;9:369-78. https:/ / doi.org/10.1016/ j.ttbdis.2017.12.001

20. Kunz C. Tick-borne encephalitis in Europe. Acta Leiden. 1992; 60:1-14.

21. Granström M. Tick-borne zoonoses in Europe. Clin Microbiol Infect. 1997;3:156-69. https:// doi.org/10.1111/j.1469-0691. 1997.tb00592.x

22. Dumpis U, Crook D, Oksi J. Tick-borne encephalitis. Clin Infect Dis. 1999;28:882-90. https://doi.org/10.1086/515195

23. Borde JP, Zajkowska J. TBE in adults. In: Dobler G, Erber W, Bröker M, Schmitt HJ, editors. The TBE Book, 2nd ed. Singapore: Global Health Press; 2019. p. 105-24.

24. Schultze D, Dollenmaier G, Rohner A, Guidi T, Cassinotti P. Benefit of detecting tick-borne encephalitis viremia in the first phase of illness. J Clin Virol. 2007;38:172-5. https://doi.org/10.1016/j.jcv.2006.11.008

25. Lotrič-Furlan S, Avsic-Zupanc T, Strle F. Is an isolated initial phase of a tick-borne encephalitis a common event? Clin Infect Dis. 2000;30:987-8. https:// doi.org/10.1086/313838

26. Saksida A, Jakopin N, Jelovšek M, Knap N, Fajs L, Lusa L, et al. Virus RNA load in patients with tick-borne encephalitis, Slovenia. Emerg Infect Dis. 2018;24:1315-23. https://doi.org/10.3201/eid2407.180059

27. van Buuren S. Multiple imputation of discrete and continuous data by fully conditional specification. Stat Methods Med Res. 2007;16:219-42. https:/ / doi.org/ 10.1177/0962280206074463

28. Perperoglou A, Sauerbrei W, Abrahamowicz M, Schmid M. A review of spline function procedures in R. BMC Med Res Methodol. 2019;19:46. https://doi.org/10.1186/ s12874-019-0666-3
29. R Core Team. R: A language and environment for statistical computing. Vienna, Austria: R Foundation for Statistical Computing [cited 2021 May 18]. http:/ / www.r-project.org

30. Lotrič-Furlan S, Bogovič P, Avšič-Županc T, Jelovšek M, Lusa L, Strle F. Tick-borne encephalitis in patients vaccinated against this disease. J Intern Med. 2017;282:142-55. https:// doi.org/10.1111/joim.12625

31. Bogovič P, Lotrič-Furlan S, Avšič-Županc T, Lusa L, Strle F. Factors associated with severity of tick-borne encephalitis: a prospective observational study. Travel Med Infect Dis. 2018;26:25-31. https:/ / doi.org/10.1016/j.tmaid.2018.10.003

32. Anić K, Soldo I, Perić L, Karner I, Barac B. Tick-borne encephalitis in eastern Croatia. Scand J Infect Dis. 1998;30:509-12. https:/ / doi.org/10.1080/00365549850161548

33. Lotric-Furlan S, Petrovec M, Avsic-Zupanc T, Strle F. Clinical distinction between human granulocytic ehrlichiosis and the initial phase of tick-borne encephalitis. J Infect. 2000;40:55-8. https:/ / doi.org/10.1053/jinf.1999.0587

34. Lotric-Furlan S, Petrovec M, Avsic-Zupanc T, Logar M, Strle F. Epidemiological, clinical and laboratory distinction between human granulocytic ehrlichiosis and the initial phase of tick-borne encephalitis. Wien Klin Wochenschr. 2002;114:636-40.

35. Lotric-Furlan S, Strle F. Thrombocytopenia, leukopenia and abnormal liver function tests in the initial phase of tick-borne encephalitis. Zentralbl Bakteriol. 1995;282:275-8. https:// doi.org/10.1016/S0934-8840(11)80127-1

36. Lotric-Furlan S, Strle F. Thrombocytopenia - a common finding in the initial phase of tick-borne encephalitis. Infection. 1995;23:203-6. https:/ / doi.org/10.1007/BF01781197

37. Saksida A, Duh D, Lotric-Furlan S, Strle F, Petrovec M, Avsic-Zupanc T. The importance of tick-borne encephalitis virus RNA detection for early differential diagnosis of tick-borne encephalitis. J Clin Virol. 2005;33:331-5. https:// doi.org/10.1016/j.jcv.2004.07.014

38. Ackermann R, Krüger K, Roggendorf M, Rehse-Küpper B, Mörtter M, Schneider M, et al. Spread of early-summer meningoencephalitis in the Federal Republic of Germany [in German]. Dtsch Med Wochenschr. 1986;111:927-33. https://doi.org/10.1055/s-2008-1068558

Address for correspondence: Petra Bogovič, University Medical Center Ljubljana, Department of Infectious Diseases, Japljeva 2, 1525 Ljubljana, Slovenia; email: petra.bogovic@kclj.si 